(C) [2009] IEEE. Reprinted, with permission, from Zheng, Luhai; Jin, Jianxun; Guo, Youguang \& Zhu, Jianguo. 2009, Design and Characteristics Analysis of LongPrimary Single-Sided Linear Induction Motor', Proceedings of IEEE International Conference on Applied Superconductivity and Electromagnetic Devices, pp. 8588. This material is posted here with permission of the IEEE. Such permission of the IEEE does not in any way imply IEEE endorsement of any of the University of Technology, Sydney's products or services. Internal or personal use of this material is permitted. However, permission to reprint/republish this material for advertising or promotional purposes or for creating new collective works for resale or redistribution must be obtained from the IEEE by writing to pubs-permissions@ieee.org. By choosing to view this document, you agree to all provisions of the copyright laws protecting it. 


\section{Design and Characteristics Analysis of Long-Primary Single-Sided Linear Induction Motor}

\author{
Luhai Zheng, Jianxun Jin \\ Center of Applied Superconductivity and Electrical Engineering \\ University of Electronic Science and Technology of China \\ Chengdu, China \\ jxjin@uestc.edu.cn, zluhai@uestc.edu.cn
}

\author{
Youguang Guo, Jianguo Zhu \\ School of Electrical, Mechanical and Mechatronic Systems \\ University of Technology Sydney, Australia \\ Sydney, Australia \\ youguang@eng.uts.edu.au
}

\begin{abstract}
Long-primary single-sided linear induction motors (SLIMs) have unique advantages in some applications such as an electromagnetic launcher with higher force density and acceleration due to its lightweight secondary conductive sheet compared with other types of LIM having different structures. Furthermore, long-primary SLIM will have superiorities without any friction loss and guidance control system when it is combined with high temperature superconducting (HTS) magnetic levitation system. In this work, a long-primary SLIM integrated with HTS levitation system is designed. This paper presents the design and performance analysis of the SLIM levitated by a HTS levitation system, and the magnetic field finite element method is applied to analysis its magnetic flux distribution, thrust force and normal force characteristics with results obtained. Finally, the preliminary motor running testing results are also presented.
\end{abstract}

Keywords-linear induction motor; high temperature superconductor; HTS magnetic levitation; electromagnetic characteristics; equivalent circuit, finite element analysis

\section{INTRODUCTION}

Based on the relative length of the primary and the secondary, single-sided linear induction motor (SLIM) may be classified into two categories, short-primary (long-secondary) and short-secondary (long-primary). Presently, short-primary SLIM is widely applied to industrial equipments and transportation systems especially in intermediate speed arrange for its lower manufacturing and operating cost [1]. However, in certain applications where higher force density or acceleration is required to be provided in short operating time, the latter would be the preferred type due to its lightweight secondary conductive sheet [2]. These applications include electromagnetic aircraft launching, car-crash testing etc.

In order to keep stable and unchangeable air-gap length, slide rails are used on both-sides of SLIM under normal circumstance, which produce sliding friction force unavoidability. Because stable levitation force can be generated between $\mathrm{NdFeB}$ permanents magnets (PMs) and high temperature superconducting (HTS) bulks [3], HTS levitation system contained PMs and HTS bulks can be combined into SLIM. The main advantage of HTS levitation system is the strong levitating force with passive and selfstabilizing feature [4], leading to the removal of the sophisticated control system for regulating the air-gap between

This work is supported by the Chinese High Technology Developmen Plan Project under Grant No. 2007AA03Z208. guideway and levitated moving secondary, which is necessary for other types of levitation transportation systems.

In this work, HTS levitation system is used in SLIM to replace the slide rails, so that the SLIM secondary rotor can move without any friction force. The correlation technique of HTS magnetic levitation system has been introduced in other papers [4-6]. In this paper, the design, electromagnetic characteristics and the operation resting results of the longprimary SLIM are introduced.

\section{MODEL OF LIM}

The model of SLIM is shown in Fig. 1, which is made up of a long stationary primary and a short moving compound secondary, and the moving secondary is levitated by the HTS magnetic levitation equipments on both sides of SLIM. Fig. 2 is the longitudinal profile of the SLIM, which shows that the primary is comprised of iron-core and embedded three-phase copper windings, and the compound secondary is consisted of aluminum ( $\mathrm{Al}$ ) sheet and back iron with the thickness of $4 \mathrm{~mm}$ and $10 \mathrm{~mm}$, respectively. Other dimensions and parameters of SLIM are shown in Table I. The HTS magnetic levitation equipments composed of YBCO HTS bulks located in low temperature vessels by the side of moving secondary on a small car and PM-guideway on both sides of primary stator. A type of PM-guideway structure is shown in Fig. 1, which is composed of PMs having the same pole in longitudinal direction and alternating poles of $\mathrm{N}$ and $\mathrm{S}$ in transverse direction, and the back iron. The levitation equipments have the function of self-levitation and self-guidance, and can make the SLIM keep the air-gap length stationary.

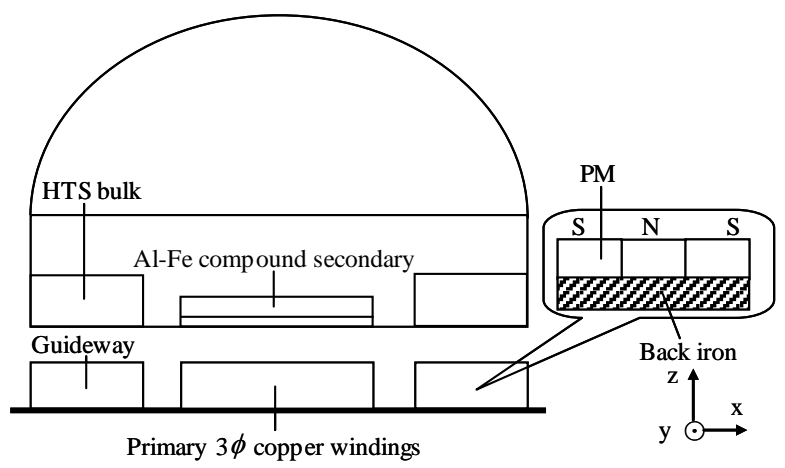

Figure 1. Model of SLIM levitated by HTS magnetic levitation system. 


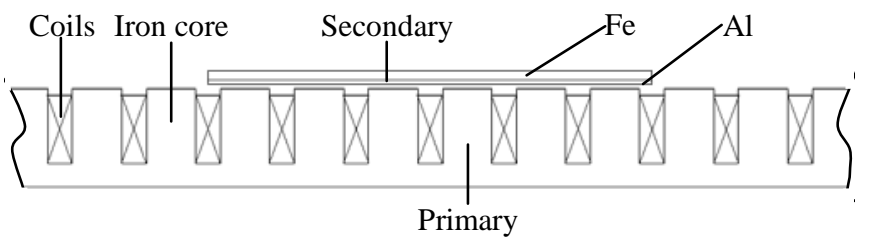

Figure 2. Longitudinal profile of long-primary SLIM.

TABLE I. MAJOR DIMENSIONS AND PARAMETERS OF SLIM.

\begin{tabular}{|l|c|}
\hline Primary & \\
\hline Copper windings & \\
\hline Number of turns & 252 \\
\hline Diameter of copper wire $(\mathrm{mm})$ & 1.2 \\
\hline Iron core & \\
\hline Tooth length (movement direction) $(\mathrm{mm})$ & 50 \\
\hline Tooth width $w_{t}(\mathrm{~mm})$ & 100 \\
\hline Slot width $w_{s}(\mathrm{~mm})$ & 25 \\
\hline Slot pitch $\lambda(\mathrm{mm})$ & 75 \\
\hline Slot depth $h_{s}(\mathrm{~mm})$ & 75 \\
\hline Pole pitch $\tau(\mathrm{mm})$ & 112.5 \\
\hline yoke height $h_{y}(\mathrm{~mm})$ & 25 \\
\hline Compound Secondary & \\
\hline Al sheet & \\
\hline Length $(\mathrm{mm})$ & 500 \\
\hline Width $(\mathrm{mm})$ & 100 \\
\hline Thickness $d(\mathrm{~mm})$ & 4 \\
\hline Back iron & \\
\hline Length $(\mathrm{mm})$ & 500 \\
\hline Width $(\mathrm{mm})$ & 100 \\
\hline Thickness $(\mathrm{mm})$ & 10 \\
\hline
\end{tabular}

\section{EQUIVALENT CIRCUIT OF LONG-PRIMARY SLIM}

For the analysis and design of a SLIM having negligible end-effects, the per-phase approximate equivalent circuit is shown in Fig. 3. The circuit components are determined from the SLIM parameters. The core losses are neglected because a realistic air-gap flux density leads to moderate flux densities in the core and hence, rather low core losses. Skin effect is small at rated frequency for a SLIM with a thin conductive sheet on the secondary. Therefore, equivalent rotor inductance is negligible. The remaining non-negligible parameters are shown in Fig. 3, and can be expressed [7,8]

$$
\begin{gathered}
X_{1}=\frac{2 \pi \mathrm{l} f\left[\left(\lambda_{s}\left(+\frac{3}{p}\right)+\lambda_{d}\right) \frac{w_{s}}{q_{1}}+\lambda_{e} l_{c e}\right] N_{1}^{2}}{p} \\
X_{m}=\frac{24 \pi u_{0} f W_{s e} K_{w} N_{1}^{2} \tau}{\pi^{2} p g_{e}} \\
\dot{I}_{2}=\frac{\dot{I}_{1}}{\sqrt{1 /(S G)^{2}+1}} \\
R_{2}^{\prime}=\frac{X_{m}}{G}
\end{gathered}
$$

where

$$
\begin{aligned}
\lambda_{s} & =\frac{h_{s}\left[1+3 k_{p}\right]}{12 w_{s}} \\
\lambda_{d} & =\frac{5\left(g_{e} / w_{s}\right)}{5+4\left(g_{0} / w_{s}\right)} \\
\lambda_{e} & =0.3\left[3 k_{p}-1\right] \\
k_{w} & =\frac{\sin (2 / m)}{q_{1} \sin \left(22 / m q_{1}\right)} \\
G & =\frac{2 \mu_{0} f \tau^{2}}{\pi\left(\rho_{r} / d\right) g_{e}}
\end{aligned}
$$

where $X_{1}$ is the per-phase stator-slot leakage reactance; $X_{m}$ the per-phase magnetizing reactance; $\dot{I}_{1}$ the rated input phase current; $\dot{I}_{2}$ the rotor phase current; $p$ the pole-pairs; $R_{2}^{\prime}$ the equivalent per-phase rotor resistance; $f$ the frequency; $q_{1}$ is the number of slots-per-pole-per-phase in the stator iron core; $k_{d}$ the distribution factor; $k_{p}$ the pitch factor, $k_{p}=\sin \left(\theta_{p} / 2\right), \theta_{p}$ the coil span in electrical degrees; $k_{w}$ the winding factor; $m$ the number of phases; $l_{\mathrm{ce}}=\theta_{p} \tau / 180^{\circ}$, $\tau$ the pole pitch; $N_{1}$ the number of turns of stator winding per phase; $W_{\text {se }}$ the equivalent stator width, $W_{\mathrm{se}}=W_{s}+g_{0} ; g_{e}$ the effective air-gap, $g_{e}=k_{c} g_{0}, k_{c}$ the Carter's coefficient, $g_{0}$ the magnetic air-gap, $g_{0}=g_{m}+d$; $S$ the slip ratio of the SLIM; $G$ the goodness factor; $\rho_{r}$ the volume resistivity of the rotor conductor outer layer, which is $\mathrm{Al}$ here; $w_{s}$ the slot width; $g_{m}$ the physic air-gap; $d$ the thickness of $\mathrm{Al}$ sheet.

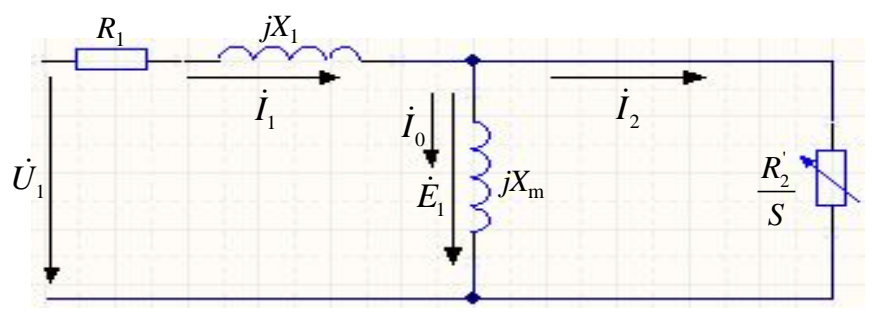

Figure 3. Per-phase SLIM equivalent circuit.

\section{CHARACTERISTICS ANALYSIS OF SLIM}

\section{A. The influence of secondary material on thrust force}

Normally, LIMs adopt compound secondary with dense conduct electricity materials used to the side of air-gap to strengthen induction current, and strong permeability magnetic materials used in another side to increase the magnetic field strength in order to enhance the electromagnetic force. The application of back iron in compound secondary is not only as the magnetic circuit, but also as part of the circuit. In rail transport field, the Al plate-back iron structure is most commonly used due to its good thrust-speed characteristics with lower cost.

Different secondary conduct electricity materials have various magnetic flux penetration-depths, and the penetrationdepth can be expressed by $\delta_{T}$ as follow 


$$
\delta_{T}=\sqrt{\frac{2}{\mu \sigma \omega}}=\sqrt{\frac{\rho}{\mu_{0} \mu_{s} \pi f}}
$$

where $\rho$ is the resistivity, $\mu_{0}$ the permeability of vacuum, $\mu_{\mathrm{s}}$ the relative permeability, $f$ the working frequency of LIM.

The relation between penetration-depth and frequency is plotted as seen in Fig. 4. It is shown that the penetration-depth decreases with the frequency quickly at low frequency and tend to stabilization above $10 \mathrm{~Hz}$, and the $\mathrm{Al}$ has relative higher penetration-depth. Fig. 5 shows the thrust force $F_{x}$ versus slip ratio $S$ characteristic for different types of compound secondary. From the Fig. 5, the Cu-Fe compound secondary have the best characteristic of thrust force $F_{X}$ secondary rotor speed $V_{r}$, due to the lower resistivity of $\mathrm{Cu}$.

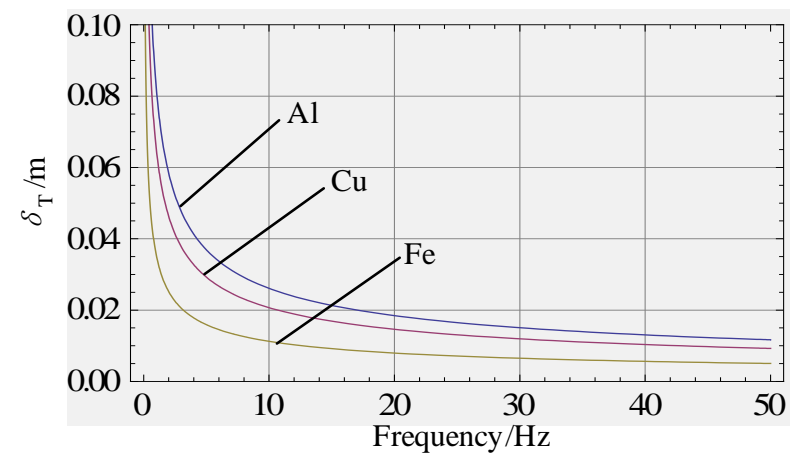

Figure 4. $\delta_{T}$ vs. frequency characteristics.

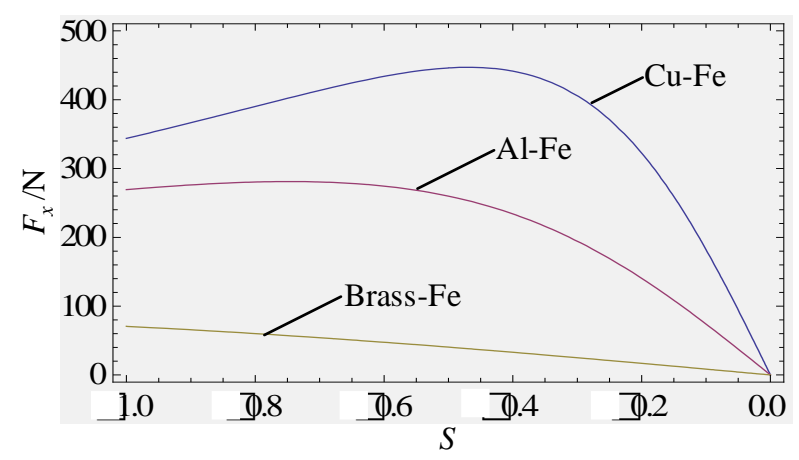

Figure 5. Thrust force vs. slip ratio for different secondary material.

\section{B. Thrust force vs velocity}

The input power to the stator windings is utilized in producing useful mechanical power which is exerted on the secondary rotor and to account for the rotor copper losses. In terms of the equivalent circuit components, the mechanical power $P_{o}$ developed by the secondary rotor is the power transferred across the air-gap from the stator to the rotor $\left(m \dot{I}_{2}^{2} R_{2}^{\prime} / S\right)$ minus the rotor copper loss $\left(m \dot{I}_{2}^{2} R_{2}^{\prime}\right)$

$$
P_{o}=m \dot{I}_{2}^{2} \frac{R_{2}^{\prime}}{S}-m \dot{I}_{2}^{2} R_{2}^{\prime}=m \dot{I}_{2}^{2} R_{2}^{\prime}\left(\frac{1-S}{S}\right)
$$

Using the equations of $P_{o}=F_{x} \cdot V_{r}$ and $S=\left(V_{s}-V_{r}\right) / V_{s}$, SLIM electromagnetic thrust $F_{X}$ generated by the SLIM stator is

$$
F_{x}=\frac{P_{o}}{V_{r}}=\frac{m I_{1}^{2} R_{2} G^{2}\left(V_{s}-V_{r}\right)}{G^{2} V_{r}^{2}-2 G^{2} V_{r} V_{S}+G^{2} V_{S}^{2}+V_{S}^{2}}
$$

where $V_{s}$ is the synchronous speed, $V_{s}=2 f \tau, V_{r}$ the secondary rotor speed. The thrust force is plotted versus varying $V_{r}$ for different working frequency as shown in Fig. 6.

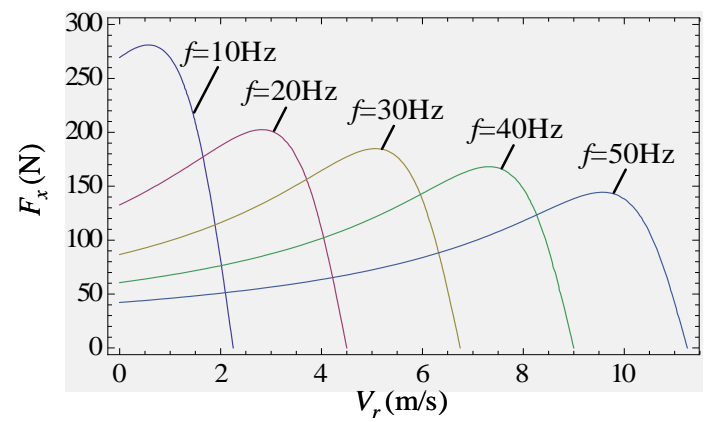

Figure 6. Thrust force $F_{X}$ vs. rotor velocity for different frequency.

\section{FINITE ELEMENT ANALYSIS OF SLIM}

Ignoring the transversal end effect, 2-D finite element (FE) model for the long-primary SLIM is built by the way of direct modeling method according to the motor geometric parameters with the magnetic vector potentials obey the periodical boundary conditions on the $y$-direction boundary lines of the primary stator and the open middle air-gap line, which is close to the practical physical model and sufficient for the theoretical analysis with low computational complexity, and the longitudinal end effect can also be considered.

The time-stepping FE method is applied in resolving, and the simulation parameters as fellows: the working frequency $f=10 \mathrm{~Hz}$, the simulation time is one period of $T=0.1 \mathrm{~s}$, the three-phase voltage: $U_{a}=165 \times \sin (\omega \mathrm{t}), \quad U_{b}=165 \times \sin (\omega \mathrm{t}-2 \pi / 3)$, $U_{c}=165 \times \sin (\omega \mathrm{t}-4 \pi / 3)$. Fig. 7 shows the three-phase symmetrical current waveforms for the windings coils after time-stepping solving with the amplitude value of $5.3 \mathrm{~A}$ and $120^{\circ}$ electrical degree differential to each other.

Fig. 8 shows the distribution of flux lines in SLIM model at one moment, and the distribution of magnetic flux in middle air-gap along the x-direction $\left(B_{x}\right)$ and y-direction $\left(B_{y}\right)$ with their sum value $\left(B_{\text {sum }}\right)$ are shown in Fig. 9.

Fig. 10 and Fig. 11 show the $F_{x}$ and $F_{y}$ versus the length of air-gap solved both by Virtual Work method and Maxwell Stress method, respectivily. It is confirmed that the $F_{x}$ and $F_{y}$ strongly depend on the length of air-gap, and both of them decrease with the air-gap length.

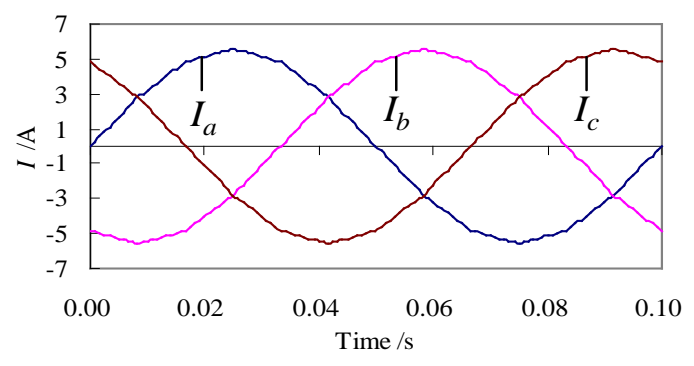

Figure 7. Three-phase current vs. time. 


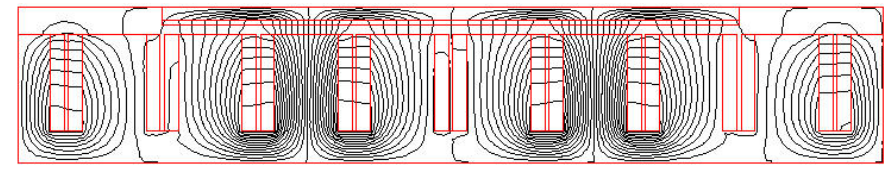

Figure 8. Distribution of Flux lines in SLIM FE model.

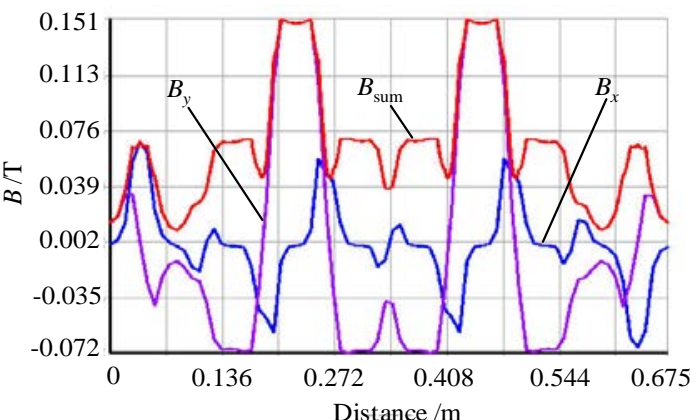

Figure 9. Distribution of magnetic flux intensity in middle air-gap.

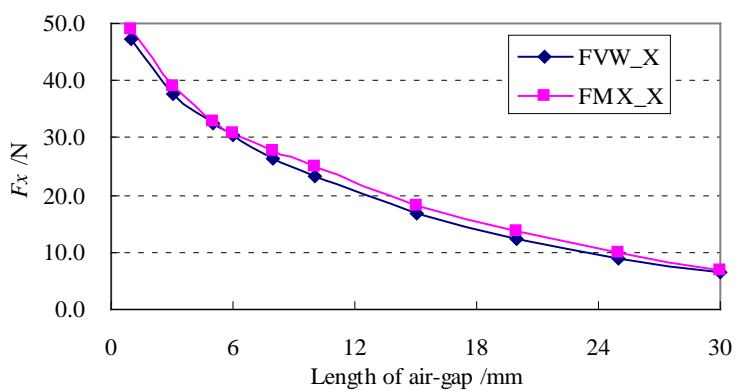

Figure 10. Thrust force $F_{x}$ vs. length of air-gap.

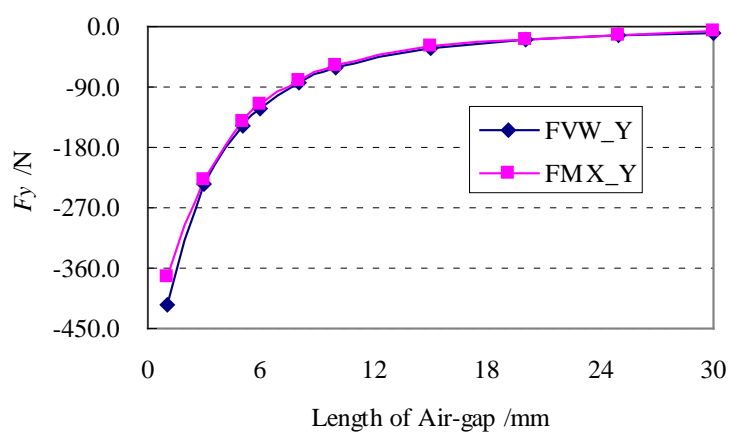

Figure 11. Normal force $F_{y}$ vs. length of air-gap.

\section{TESTING RESUlts}

The converter based on the Voltage Space Vector PWM (SVPWM) strategy was applied in the control of the SLIM. Fig. 13 shows the amplitude value of phase current variation with running frequency, which indicates the current decrease with the frequency. Fig. 14 shows the thrust force versus frequency characteristics for different slip ratios. It is observed that the $F_{x}$ increases with the frequency (less than $50 \mathrm{~Hz}$ ) slowly during the low slip ratio less than 0.1 , and increases with frequency and then decreases with frequency when slip ratio above 0.2 . As can be seen from the chart, the lower slip ratio will have better $F_{X}$ vs frequency characteristic during the high frequency applications.

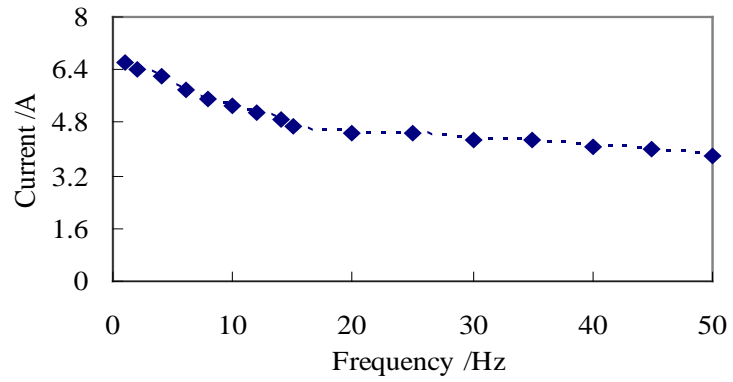

Figure 12. Current vs. frequence.

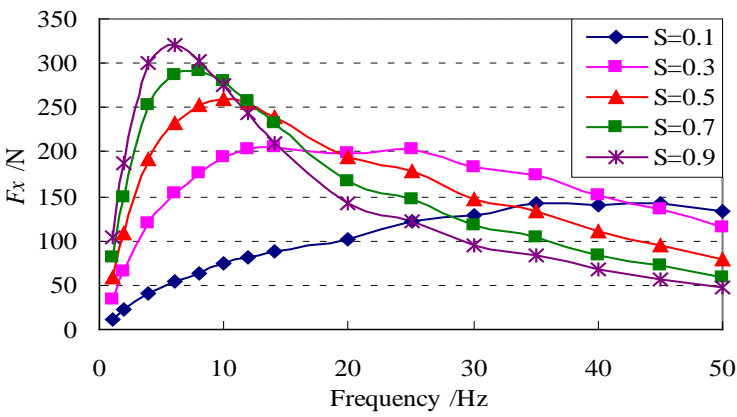

Figure 13. Thrust force vs. frequency for different $S$.

\section{CONCLUSION}

This paper presents the design of a long-primary SLIM. The equivalent circuit of SLIM has been built with correlative characteristics described. Magnetic field finite element method is applied to analyze the force characteristics of the SLIM, and the primary running testing results are obtained. The results show that the Cu-Fe compound secondary have better thrustspeed characteristic, and the length of air-gap has a great influence on thrust force. In high-frequency applications, the SLIM would have relative better thrust-velocity performance.

\section{REFERENCES}

[1] T. Onuki, Y. Kamiya, Y. Kurimoto, and M. Yoshizawa, “A novel block feeding method in the single-sided linear induction motor with a short secondary member,” IEEE trans. Energy Convers., vol. 14, no. 4, pp. 1323-1328, 1999.

[2] J. X. Jin, Y. G. Guo, and J. G. Zhu, "Principle and analysis of a linear motor driving system for HTS levitation applications,” Physica C, vol. 460-462 II, pp. 1445-1446, 2007.

[3] H. Fukai, M. Tomita, M. Murakami, and T. Nagatomo, "Numerical simulation of trapped magnetic field for bulk superconductor,” Physica C, vol. 357-360, pp. 774-776, 2001.

[4] J. X. Jin and L. H. Zheng, "Verification of levitation force between a HTS and a permanent magnet,” Phys. C,vol.460-462,pp.1457-1458,2007.

[5] J. X. Jin and L. H. Zheng, "Performance analysis and optimization design of a HTS LSM,” J. of Sci., Tech. and Eng., vol. 2, no. 1, pp. 1922, 2008.

[6] J. X. Jin, L. H. Zheng, and Y. G. Guo, "HTS levitated mobile technology and prototype,” The 2007 IEEE Inter. Conf. on Integration Tech., pp. 47-51, March 20-24, 2007, Shenzhen, China.

[7] R. M. Pai, I. Boldea, and S. A. Nasar, "A complete equivalent circuit of a linear induction motor with sheet secondary,” IEEE Trans. Magn., vol. 24, no. 1, pp. 639-654, 1988.

[8] M. Assadollahbaik, "Linear Induction motor for pumping capsules in pipes,” Ph.D. Dissertation, Department of Civil Engineering, University of Missouri- Columbia, July 1984. 